\title{
RECICLAJE DE RESIDUOS CAFETALERO, DESCOMPOSICIÓN Y LIBERACIÓN DEL NITRÓGENO
}

\author{
RECICLAGEM DE RESÍDUOS DE CAFEZAL, \\ DECOMPOSIÇÃO E LIBERAÇÃO DE NITROGÊNIO.
}

\begin{abstract}
Alberto Pérez Díaz ${ }^{1 *}$, Sâmia Maria Tauk-Tornisielo ${ }^{2}$, Carlos Bustamante González ${ }^{3}$, Gloria Marta Martín Alonso ${ }^{4}$, Ramón Rivera Espinosa ${ }^{4}$, Rolando Viñals Nuñez ${ }^{3}$, Victor Alvarez Villar $^{1}$
\end{abstract}

${ }^{1}$ Universidad de Guantánamo. Ave. Che Guevara, Km 2 1⁄2, Municipio Guantánamo, provincia Guantánamo, Cuba, CP 95 300. E-mail: albertopd@ @ug.co.cu.

${ }^{2}$ Centro de Estudos Ambientais, Universidade Estadual Paulista - UNESP, Campus de Rio Claro, avenida 24-A, 1515, Bela Vista, CEP 13506-900. Rio Claro, SP, Brasil. seb@rc.unesp.br.

${ }^{3}$ Instituto de Investigaciones Agroforestales, UCTB Tercer Frente, CP 92700,

Santiago de Cuba, Cuba. E-mail: bustamante@ecicc.ciges.inf.cu.

${ }^{4}$ Instituto Nacional de Ciencias Agrícolas. Carretera de Tapaste, Km 3 1/2, Gaveta

Postal No 1, San José de las Lajas, Mayabeque, Cuba Código Postal 32700 E-mail:

gloriam@inca.edu.cu, rrivera@inca.edu.cu.

*Autor para correspondência. E-mail: albertopd@cug.co.cu.

\begin{abstract}
RESUMEN
La investigación se desarrolló durante el período de nueve años en dos localidades cafetaleras de Cuba, para evaluar la fitomasa, el $\mathrm{N}$ reciclado y la velocidad de descomposición de diferentes componentes del agroecosistema. Se determinaron la masa seca y el contenido de $\mathrm{N}(\%)$ y los datos se expresaron como kg. ha ${ }^{-1} \cdot \mathrm{mes}^{-1}$. Para estudiar la velocidad de descomposición de los restos vegetales, se utilizó el método de las bolsas, en cada bolsa se depositó el material vegetal en específico a estudiar (50 g de masa fresca) y se distribuyeron de forma homogénea. Se encontró que los aportes de biomasa de restos de los árboles de sombra fueron mayores que la caída de sus hojas, todos ellos con importantes retornos de $\mathrm{N}$ al suelo. Los restos tuvieron inicialmente un proceso de inmovilización del $\mathrm{N}$ y posteriormente comenzaron a liberar lo mismo de su biomasa, pero su magnitud dependió del material evaluado. Se encontraron diferencias en la tasa de descomposición de los residuos dentro del agroecosistema cafetalero, con un tiempo de vida media $\left(\mathrm{t}_{1 / 2}\right)$ que varió entre las especies.
\end{abstract}

Palabras-clave: Tasa de Descomposición. Agroecosistema. Cafetalero. Tasa de Nitrógeno 


\section{RESUMO}

O estudo se desenvolveu durante o período de nove anos em duas localidades de cafezáis em Cuba para quantificar a fitomassa, nitrogênio reciclado e a velocidade de decomposição de diferentes componentes do agroecossistema. Foram determinados a massa seca e o teor de $\mathrm{N}(\%)$ e os valores foram expressos em $\mathrm{kg}$. ha ${ }^{-1} \cdot \mathrm{mes}^{-1}$. Para se estudar a velocidade de decomposição dos restos vegetais, foi usado o método de bolsas. Em cada bolsa foi colocado material vegetal específico deste estudo ( $50 \mathrm{~g}$ de massa fresca) e colocado de forma homogénea. Foi observado que o aporte de biomassa dos restos das árvores de sombra foi maior do que a quantidade de folhedo, mas todos estes resíduos contribuíram para importante retorno de $\mathrm{N}$ ao solo. Houve inicialmente um proceso de imobilização do $\mathrm{N}$, mas posteriormente este começou a ser liberado da biomassa, contudo, a quantidade dependeu do material estudado. Foram observadas diferentes taxas de decomposição dos residuos dentro dos cafezais, tendo um tempo de vida média $\left(t_{1 / 2}\right)$ que variou entre as espécies utilizadas.

Palavras-chave: Taxa de Decomposição. Agroecossistema. Cafezais. Taxa de Nitrogênio.

\section{INTRODUCCIÓN}

El estudio del ciclo de nutrientes en los agroecosistemas es fundamental para comprender la dinámica de los elementos y su disponibilidad en un manejo sostenible del sistema productivo (CARDONA, 2004; CARDONA y SADEGHIAN, 2005). Los avances alcanzados son significativos en la comprensión del transporte de nutrimentos dentro de los cafetales a libre exposición y en asociación con árboles de sombra dentro del agroecosistema cafetalero (GARBISU et al., 2007).

El cafetal es un sistema cerrado y las investigaciones deben estar dirigidas a conocer todo el potencial del mismo para tratar de utilizar todas sus posibilidades. Entre los eslabones de este ecosistema se encuentran los árboles de sombra, los residuos del control de arvenses y de la cosecha, los materiales utilizados como cobertura y abono verde (ARELLANO et al., 2004).

La descomposición es proporcional al contenido de materia orgánica y a la tasa relativa de descomposición o transferencia de material vegetal mensual hacia el suelo y puede ser representada por modelos exponenciales simples (JIMÉNEZ et al., 2005).

La hojarasca juega un papel fundamental en el ciclo de nutrientes y en la transferencia de energía entre las plantas y el suelo, especialmente en agroecosistemas desarrollados en suelos pobres, donde la producción depende del aporte de nutrientes a través del proceso de descomposición. Una caída consistente de hojarasca a lo largo del año, acoplado con una lenta descomposición, puede proteger a los suelos contra la erosión (PHILPOTT et al., 2006).

Las investigaciones en los agroecosistemas cafetaleros en Cuba, se enfocaron en caracterizar los aspectos más apremiantes: obtención de dosis; fraccionamiento más adecuado, el establecimiento de métodos de diagnóstico (PEREZ et al., 2014a), la participación del $\mathrm{N}$ del fertilizante y del suelo en la nutrición del cafeto (PÉREZ et al., 2011) y su efecto sobre la calidad de los suelos (PEREZ et al., 2014b). En esta investigación el objetivo fue la determinación de los aportes y transferencia de nutrientes de los componentes del agroecosistema.

\section{MATERIALES Y MÉTODOS}

La investigación se desarrolló durante nueve años en dos localidades cafetaleras de Cuba: Tercer Frente, situada en el municipio Tercer Frente, $20^{\circ} 09^{\prime}$ latitud Norte y $76^{\circ} 16^{\prime}$ longitud Oeste, a 150 m.s.n.m. en la provincia Santiago de Cuba y La Alcarraza está situada en el municipio Sagua 
de Tánamo, 20³5' latitud Norte y $75^{\circ} 15^{\prime}$ longitud Oeste, a 300 m.s.n.m de la ciudad de Holguín, provincia Holguín, Cuba.

\subsection{Evaluación de la fitomasa $y$ el $\mathbf{N}$ reciclado por la hojarasca de los árboles de sombra y el cafeto}

La cuantificación de la caída de la fitomasa del cafeto (hojas) y de los árboles de sombra (hojas, frutos, flores y raquis) al suelo del agroecosistema cafetalero se realizó mensualmente desde enero a diciembre en las dos localidades y durante los dos ciclos productivos del cafeto robusta. La poda del cafeto se realizó en febrero del primer año. El retorno de los deshijes, otros restos de cafeto (flores, frutos y ramas) y las arvenses se realizó durante tres años.

La estimación de la cantidad de hojarasca caída de los árboles de sombra en cada localidad se realizó por medio de mantas de nylon de poliesturano de $1 \mathrm{~m}$ x $1 \mathrm{~m}$. Se seleccionaron al azar en cada área experimental, cuatro árboles de sombra y se colocaron 8 mantas por árbol, cuatro en la calle y 4 en la hilera del cafeto (PÉREZ y SOZA, 2006). Cada mes se procedió a reunir todo el material recolectado en cada manta y posteriormente se limpiaron los restos de suelo y se separaron por especie y componente en hojas, ramas, flores y raquis.

Se determinaron la masa seca, el contenido de N (\%) y se expresaron como $\mathrm{kg}^{\mathrm{h}} \mathrm{ha}^{-1}$.mes 1. Se estimó además el aporte de fitomasa y $\mathrm{N}$ asociado que representaron la poda y los deshijes que se realizaron al cafeto robusta.

Para cuantificar el aporte de la poda y los deshijes, se tomaron al azar las muestras de cinco plantas en el tratamiento del sistema de fertilización $\mathrm{N}_{2}$. Para la poda se colectaron los tallos y ramas de las plantas. La práctica del deshije se realizó tres veces por año según lo recomendado por DÍAZ et al. (2007).

Para la estimación de las cantidades de fitomasa $\left(\mathrm{t} \mathrm{ha}^{-1}\right)$ y de las cantidades de $\mathrm{N}$ asociado $\left(\mathrm{kg} \mathrm{ha}^{-1}\right)$ se calculó el área neta por hectárea $(\%)$ correspondiente a las arvenses, descontando las áreas vitales de los árboles de sombra y de los cafetos. Se identificaron por especies (Tabla 1) y se les determinó su porcentaje de participación en la mezcla mediante la fórmula:

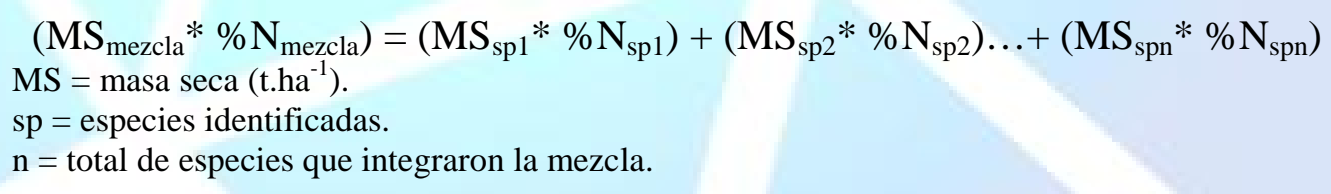

\subsection{Descomposición de la hojarasca del cafeto y los árboles de sombra}

Para estudiar la velocidad de descomposición de los restos vegetales, se utilizó el método de las bolsas (ANDERSON y INGRAM, 1996) en el sitio Tercer Frente durante tres año. 
Tabla 1 - Identificación de la mezcla de arvenses presentes en los agroecosistemas cafetaleros estudiados. Frecuencia de aparición en las limpias. Tercer Frente - (Tercer Frente, Santiago de Cuba), Cuba.

\begin{tabular}{|l|l|c|}
\hline \multicolumn{1}{|c|}{$\begin{array}{c}\text { Nombre } \\
\text { vulgar }\end{array}$} & \multicolumn{1}{|c|}{ Nombre científico } & $\begin{array}{c}\text { \% aparición en las } \\
\text { limpias }\end{array}$ \\
\hline Ají Culebra & Rivina humilis Lin. & 92,9 \\
\hline Amor Seco & Priva lappulacea, Lin. & 50,0 \\
\hline Anamú & Petiveria alliacea, Lin. & 78,6 \\
\hline Canutillo & Comnelina elegans, H.B. K. & 64,3 \\
\hline Cañamazo & $\begin{array}{l}\text { Stenotaphrum secundatum, } \\
\text { Walt. }\end{array}$ & 85,7 \\
\hline Caramana & Cyperus rotundum, Lin. & 78,6 \\
\hline Dormidera & Mimosa pudica, Lin. & 57,1 \\
\hline Empanadilla & $\begin{array}{l}\text { Desmodium adscendens, } \\
\text { P.D.C. }\end{array}$ & 71,4 \\
\hline Garro Blanco & Spermacoce tenuior, Lam. & 78,6 \\
\hline Lespedeza & Lespedeza polytachya, Michx & 85,7 \\
\hline Leucaena & Leucaena glauca, Lin. & 71,4 \\
\hline Malva & Bastardia viscosa, Lin & 92,9 \\
\hline Pata De Gallina & $\begin{array}{l}\text { Dactyloctenium aeggytium, } \\
\text { Lin. }\end{array}$ & 50,0 \\
\hline Sanguinaria & $\begin{array}{l}\text { Achyranthes poligonoydes, } \\
\text { Lin. }\end{array}$ & 100,0 \\
\hline Súrbana & Panicum fasciculatum, SW. & 100,0 \\
\hline Zorra & Tricholaena rosea, Nees. & 71,4 \\
\hline
\end{tabular}

Para cada uno de los residuos se prepararon 24 bolsas de malla de polietileno de $20 \mathrm{~cm}$ x 15 cm con rejillas de $1 \mathrm{~mm}$ x $1 \mathrm{~mm}$. En cada bolsa se depositó el material vegetal en específico a estudiar (50 g de masa fresca) y se distribuyeron de forma homogénea en todo el área de la bolsa, procurando que no quedaran compactas, para facilitar la descomposición y simular el estado en que quedan naturalmente en la superficie del suelo después de su caída. Las bolsas se distribuyeron aleatoriamente en la plantación, debajo de los cafetos y se colocaron en el mes de marzo de cada año.

Se estudió la descomposición de diferentes residuos, para cada uno de los cuales se realizó un ensayo específico. Los residuos estudiados fueron los siguientes:

- Hojas de cafetos: se utilizaron hojas recién caídas.

- Hojarasca de algarrobo: compuesta por hojas, flores y vainas caídas.

- Hijos del café: se tomaron al momento de realizar los deshijes.

- Arvenses: se tomaron de la mezcla de las especies encontradas en el área en el momento de realizar la limpia.

Las evaluaciones se realizaron mensualmente hasta los 210 días, durante tres años . Se recolectaron mensualmente 3 bolsas para su evaluación. Al material remanente se le eliminaron los residuos de suelo y de otras especies; se secaron a $70^{\circ} \mathrm{C}$ hasta alcanzar valores de peso constantes mediante una balanza analítica.

Para evaluar la descomposición de los materiales vegetales se determinó la masa seca remanente (MSR) en las bolsas en cada momento de muestreo y se expresó como porcentaje de la masa seca inicial.

$$
\operatorname{MSR}(\%)=\frac{\text { masa seca muestreo }(\mathrm{g})}{\text { masa seca inicial }(\mathrm{g})} \text { 100 }
$$


La determinación de la tasa de descomposición del material vegetal, se estimó a través de un modelo exponencial simple del orden:

$$
\mathrm{y}=\mathrm{y}_{0} \mathrm{e}^{-\mathrm{kt}}(\mathrm{JIMÉNEZ} \text { et al., 2005) }
$$

$\mathrm{y}=$ porcentaje de masa seca remanente.

$\mathrm{y}_{0}=$ porcentaje de masa seca inicial.

$\mathrm{t}=$ tiempo.

$\mathrm{k}=$ tasa relativa de descomposición mensual o constante de velocidad de descomposición.

El coeficiente de descomposición $(\mathrm{k})$ indica la velocidad con que se transforman o descomponen los residuos vegetales, y se determinó por la fórmula (JIMÉNEZ et al., 2005):

$$
\mathrm{k}=-\left[\ln \left(\mathrm{y} / \mathrm{y}_{0}\right)\right] / \mathrm{t}
$$

Se determinó la concentración de nitrógeno $(\% \mathrm{~N})$ en la masa seca remanente de los diferentes residuos vegetales y de cada una de las bolsas mediante el método colorimétrico. El flujo o transferencia de nitrógeno liberado mensualmente se determinó por la fórmula descrita por JIMÉNEZ et al. (2005):

$$
\% \mathrm{~N} \text { liberado }=100-[(\mathrm{Nm} / \mathrm{Ni}) * \mathrm{MSR}]
$$

$\% \mathrm{~N}$ liberado $=$ porcentaje del nutrimento liberado mensualmente.

$\mathrm{Nm}=$ concentración de nutrimento en la muestra residual mensual $(\%)$.

$\mathrm{Ni}=$ concentración del nutrimento en la muestra inicial (\%).

MSR = masa seca remanente $(\%)$.

El tiempo medio de vida de la materia seca y del nutriente en el residuo vegetal, o lo que es lo mismo, el tiempo necesario para que la mitad de ese residuo desaparezca o para que el $\mathrm{N}$ se libere, se calculó por la ecuación:

$$
\mathrm{t}_{1 / 2}=\ln (2) / \mathrm{k}(\text { GOURETE, 2007). }
$$

En todas las evaluaciones realizadas se determinó la media y la desviación estándar para cada tipo de material en estudio.

\section{RESULTADOS Y DISCUSIÓN}

\subsection{Reciclaje de hojas y otros componentes del agroecosistema}

En el primer año de establecimiento del cafeto la planta se encuentra en una etapa de crecimiento vegetativo (aéreo y radical), no estando presente la formación de frutos el cual condiciona entre otros aspectos la caída de las hojas. Es por eso que, en el primer ciclo, el retorno por caída de hojas, apareció a partir del segundo año con valores de 0.30 t.ha $^{-1}$ de masa seca, correspondiente al año del despunte (Figura 1). 


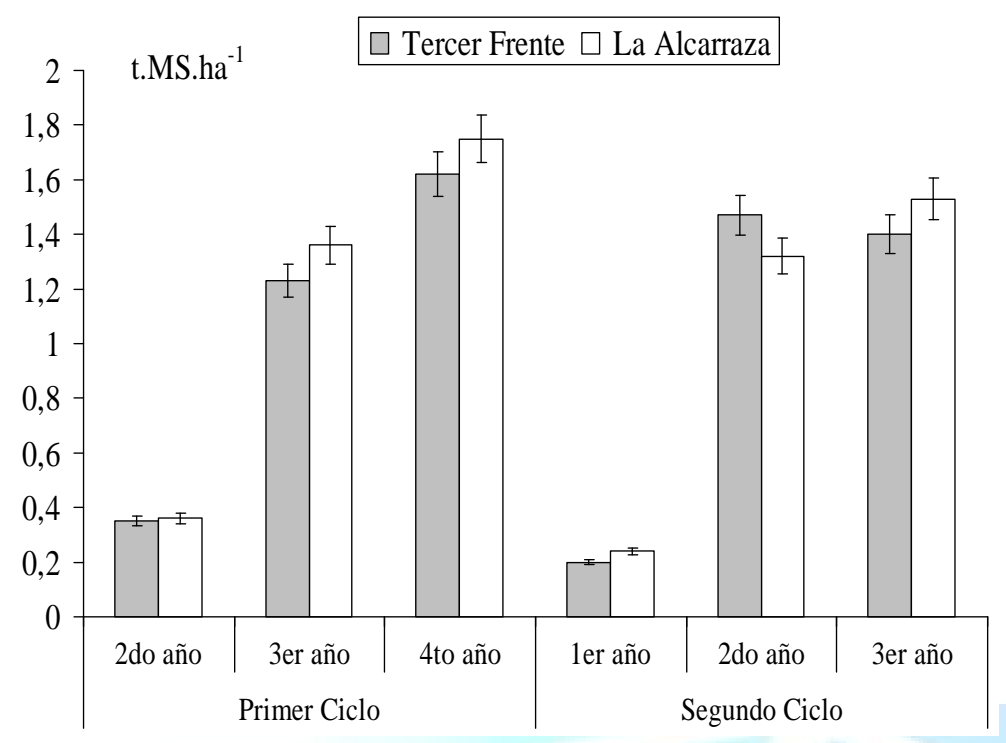

Figura 1. Aporte de biomasa (t.ha ${ }^{-1}$ de masa seca) por las hojas de cafeto. Barras verticales: desviación estándar de las medias. Tercer Frente - (Tercer Frente, Santiago de Cuba) y La Alcarraza (Sagua de Tánamo, Holguín), Cuba.

Al pasar a la etapa propiamente productiva, la caída de hojas se incrementó en ambos sitios, y su magnitud estuvo en dependencia de la cosecha del año en cuestión; así en el tercer año, el retorno de las hojas representó del $24-26 \%$ del total de las hojas de la planta, incrementándose en el cuarto año $(30-32 \%$ del total), correspondiente con altas cosechas del cafeto robusta.

Como el cafeto después de la poda adelanta en un año la entrada en producción, se encontraron retornos iniciales de caída de hojas en el orden de $0.20-0.24$ t.ha ${ }^{-1}$ de masa seca en el primer año del segundo ciclo productivo. La caída de hojas en el segundo y tercer año después de la poda fue superior a la obtenida en igual período del ciclo anterior, con valores que representaron el $33 \%$ del total, pero siempre relacionada con las altas cosechas, encontradas en los primeros años de este segundo ciclo respecto al anterior y asociadas con un mayor nivel de precipitaciones.

La incorporación de otros restos del cafeto (flores, frutos y ramas) al agroecosistema estuvo en el orden de 0,2 a 0,3 t.ha ${ }^{-1}$ de masa seca (Figura 2 A). Por concepto de la poda de los cafetos, además de las hojas, se incorporaron importantes cantidades de materiales en forma de ramas y los tallos en el año que se realizó la misma. La producción de biomasa (incluye todos los materiales) en dicho año estuvo en el rango de 5.29 a 5.63 t.ha $^{-1}$ de masa seca (Figura 2 B), mientras que por los diferentes órganos del cafeto evaluados se retornó $0.8-0.92$ t.ha $^{-1}$.año ${ }^{-1}$ de masa seca en el periodo evaluado.

Otro de los componentes que contribuyen al aporte de biomasa en el agroecosistema cafetalero, lo constituyó el deshije. La contribución por el retorno de esta práctica dentro del manejo de Coffea canephora se incrementó por año (Figura $2 \mathrm{C}$ ).

Por lo que, con la realización de al menos tres deshijes por año se pueden incorporar de 0.30 a 0.8 t.ha ${ }^{-1}$ de masa seca. La media de los años evaluados permite un retorno de $0.54 \mathrm{t}^{\text {. ha }}{ }^{-1}$. año ${ }^{-1}$ en Tercer Frente.

La fitomasa producida por las arvenses dependerá, entre otros factores, del manejo de la sombra y del desarrollo del cafeto dentro del área. Así, la realización de cuatro limpias anuales de las arvenses y el mantenimiento de un $30 \%$ de la sombra en Coffea canephora, retornó en el año de la poda valores de 1.80 t.ha $^{-1}$ de masa seca. 


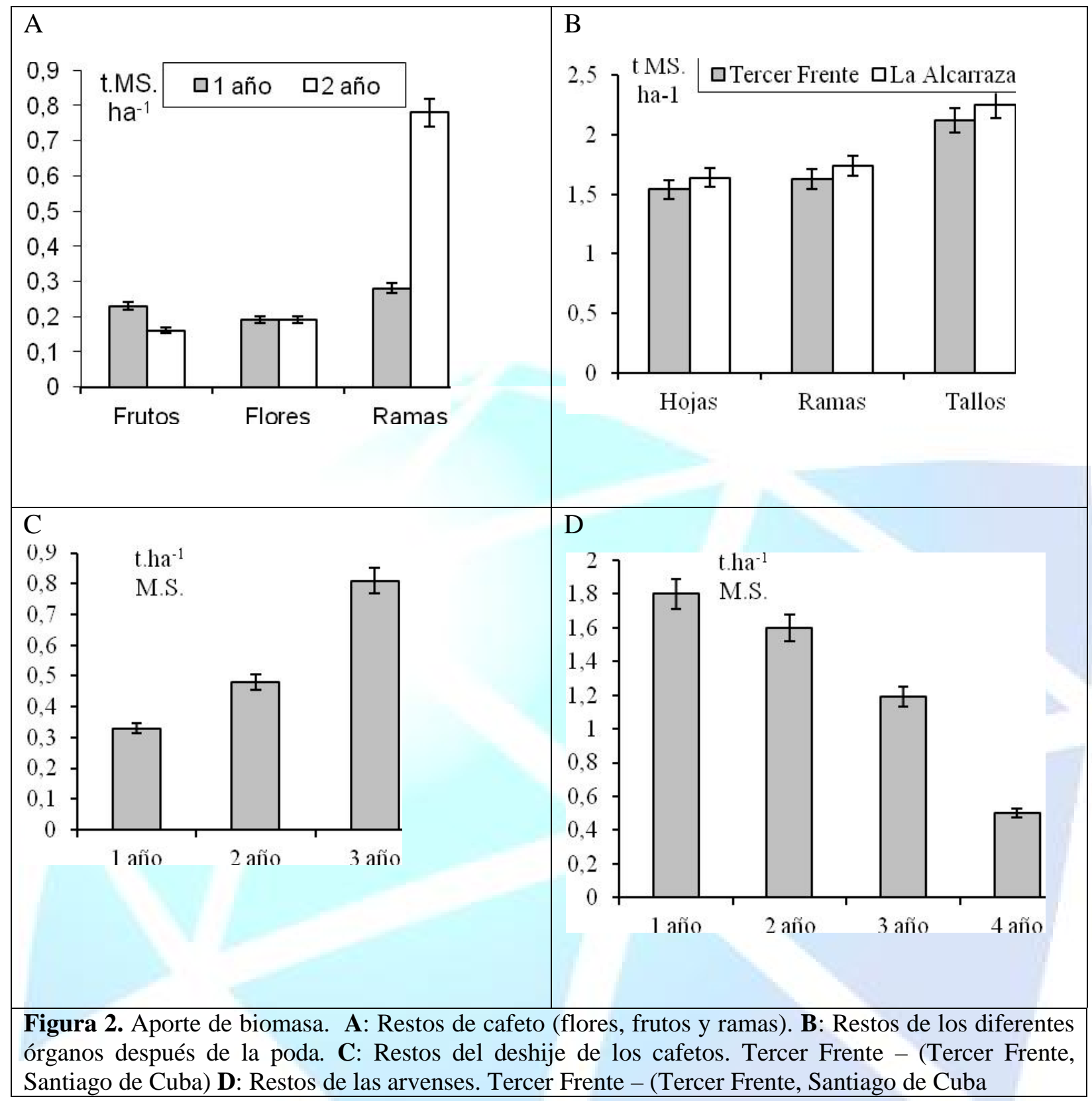

Los retornos realizados en el segundo y tercer años fueron de 1.19 t.ha $^{-1}$ a 1.6 t.ha $^{-1}$ de masa seca (Figura 2 D), mientras que en el cuarto años se redujo a 0.50 t.ha $^{-1}$ de masa seca. Estos resultados fueron similares a lo alcanzado por Quintero y Ataroff (1998) en Venezuela, con aportes de biomasa por el deshierbe de 1.9 t.ha $^{-1}$. año ${ }^{-1}$ de masa seca.

\subsection{Contenido de $\mathbf{N}$ de las hojas y otros componentes del cafeto $\mathbf{y}$ los árboles de sombra}

Las hojas del cafeto retornaron importantes cantidades de $\mathrm{N}$ al suelo, desde $6.1 \mathrm{~kg}^{-h^{-1}}$ en el segundo año de establecimiento del cafeto (año de menor caída de hojas), 40 - $45 \mathrm{~kg} \cdot \mathrm{ha}^{-1}$ en el tercer año y $46-48 \mathrm{~kg} \cdot \mathrm{ha}^{-1}$ de $\mathrm{N}$ en el cuarto año (Figura 3 ).

En el segundo ciclo, los retornos de $\mathrm{N}$ comenzaron desde el primer año y llegaron en el tercer año a $38 \mathrm{~kg} \cdot \mathrm{ha}^{-1}$. Como media, los retornos anuales de $\mathrm{N}$ por la caída de las hojas de Coffea canephora durante los años evaluados fueron del orden de los $30 \mathrm{~kg} \cdot \mathrm{ha}^{-1} \cdot$ año $^{-1}$ 
Los restos del cafeto retornaron por medio de los frutos $4.96 \mathrm{~kg} \cdot \mathrm{ha}^{-1} \cdot \mathrm{año}^{-1}$; por las flores, $2.09 \mathrm{~kg} \cdot \mathrm{ha}^{-1} \cdot \mathrm{año}^{-1}$ y las ramas contribuyeron con $10.50 \mathrm{~kg} \cdot \mathrm{ha}^{-1} \cdot \mathrm{año}^{-1} \mathrm{de} \mathrm{N}$; mientras que los restos del algarrobo incorporaron $48.14 \mathrm{~kg} \cdot \mathrm{ha}^{-1} \cdot \mathrm{anno}^{-1}$ de $\mathrm{N}$ y los del piñón $44.09 \mathrm{~kg} \cdot \mathrm{ha}^{-1} \cdot \mathrm{año}^{-1}$ de $\mathrm{N}$ (Figura 4A).

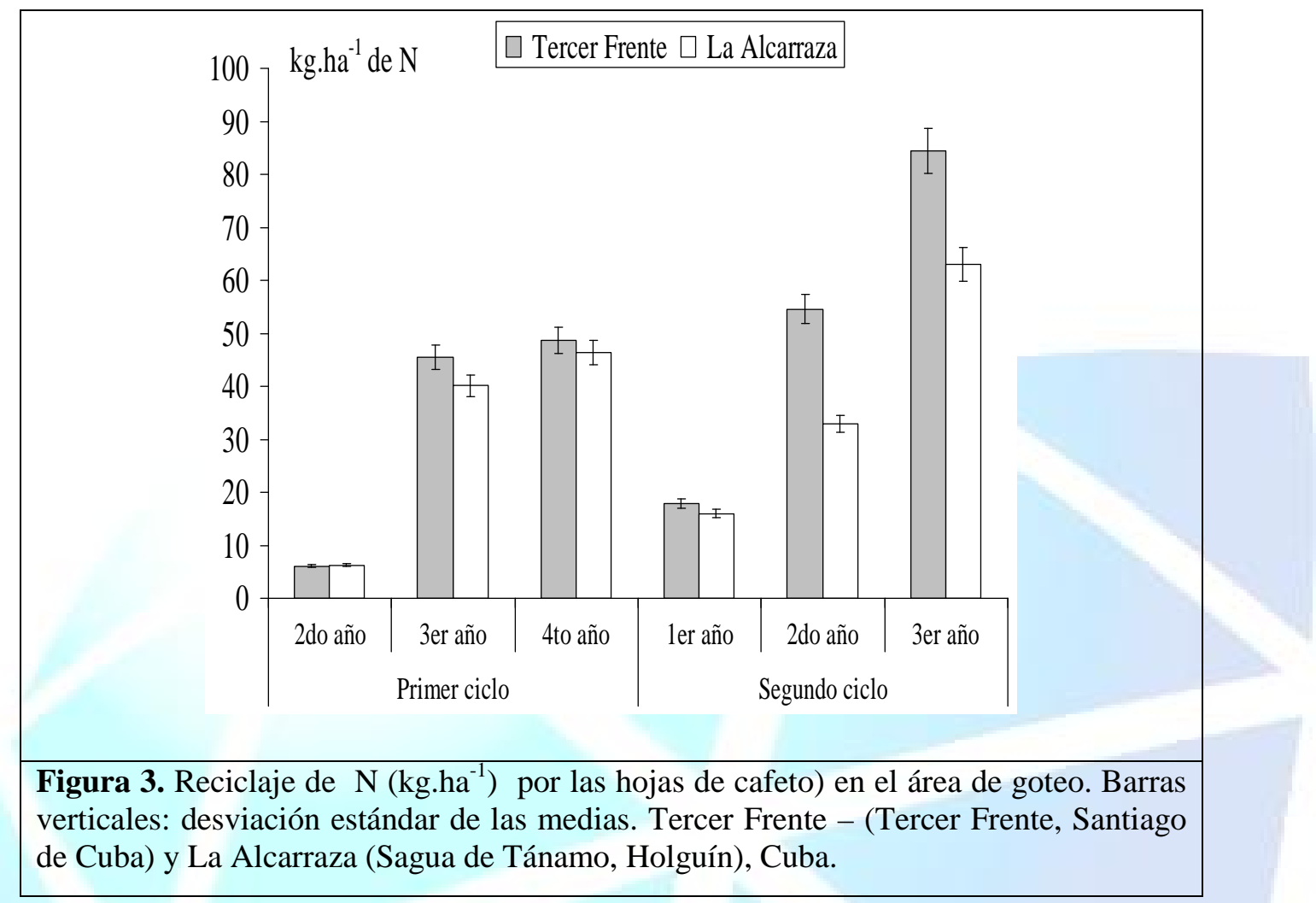

Por el deshije del cafeto se incorporaron al suelo entre $10.4-25.45 \mathrm{~kg}^{-h a^{-1}}$ de N (Figura 4 C). Como media de las evaluaciones realizadas, por este concepto se aportarían $18.42 \mathrm{~kg} \cdot \mathrm{ha}^{-1} \cdot$ año $^{-1}$ (sitio Tercer Frente). Estos resultados fueron similares a los encontrados en Venezuela, donde los deshijes del cafeto aportaron como valores medios $19.6 \mathrm{~kg} \cdot \mathrm{ha}^{-1}$ año ${ }^{-1} \mathrm{de} \mathrm{N}$ (QUINTERO y ATAROFF, 1998). Por efecto de la poda total se incorporaron $44.13-50.5 \mathrm{~kg}^{-\mathrm{ha}^{-1}}$ por las hojas del cafeto, por los tallos de $21.37-22.5 \mathrm{~kg} \cdot \mathrm{ha}^{-1}$ y por las ramas de $18.89-20.7 \mathrm{~kg} \cdot \mathrm{ha}^{-1}$ de N. 


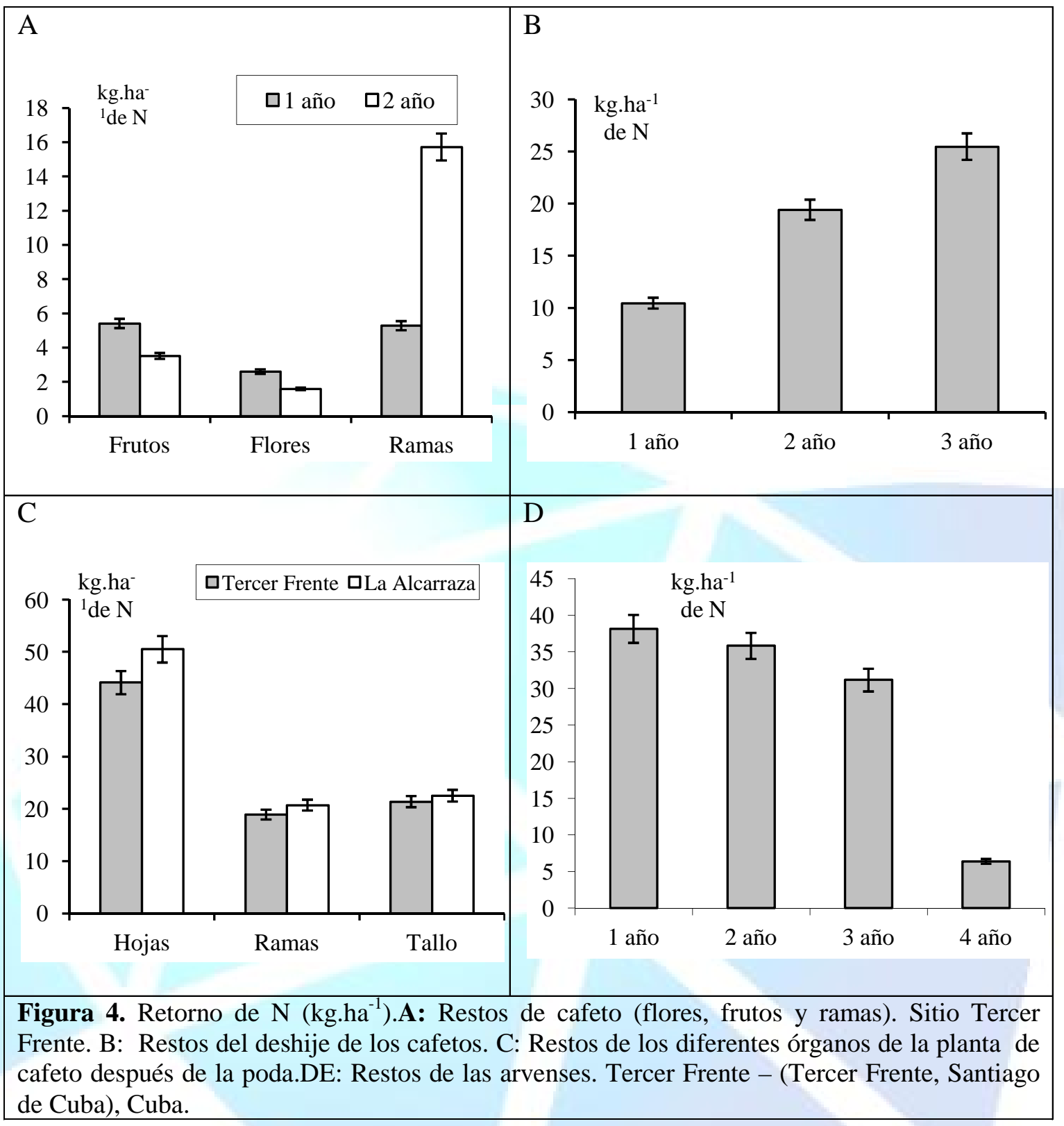

El deshierbe permite el retorno de toda la biomasa aérea de las especies que conforman el estrato herbáceo del cafetal y con ella, el N, el cual tuvo un comportamiento diferenciado por año, disminuyendo en la medida que las plantas de cafeto crecen y cierran las calles. Los mayores contenidos del $\mathrm{N}$ se encontraron en el año de la poda $\left(38 \mathrm{~kg} \cdot \mathrm{ha}^{-1}\right)$, y disminuyó con el tiempo hasta el cuarto año, con sólo valores de $6 \mathrm{~kg} \cdot \mathrm{ha}^{-1}$ de N (Figura $4 \mathrm{D}$ ).

\subsection{Tasa de descomposición de los diferentes componentes del agroecosistema}

La tasa de descomposición varió por cada material y se incrementó con el tiempo, así las arvenses fueron las más rápidas en descomponerse con una masa seca remanente (MSR) a los 210 días de sólo $17 \%$, seguida del algarrobo con $33 \%$ (Figura 5). Las arvenses presentaron una favorable relación $\mathrm{C} / \mathrm{N}$ de 12.20 , influenciada tal vez por la propia mezcla de especies existente en el agroecosistema; mientras que la hojarasca del algarrobo tuvo una relación $\mathrm{C} / \mathrm{N}$ de 13.43 muy apropiada para una rápida descomposición. 
La tasa de descomposición encontrada en el algarrobo fue similar a lo reportado por Gourete (2007), donde la hojarasca de las especies Erythrina sp. e Inga sp. presentaron las menores masas remanentes a partir de los 150 días, y llegaron a ser alrededor del $30 \%$.

La tasa de descomposición de los restos de los deshijes (relación $\mathrm{C} / \mathrm{N}$ de 30.2 ) fue la más lenta dentro de los materiales evaluados; así a los 210 días quedaba el $56 \%$ de la MSR. El hecho que los deshijes tuvieran menor tasa de descomposición que le resto de los materiales estudiado, se debe a que, normalmente las hojas presentan mayor tasa de descomposición, en razón de su concentración inicial más elevada de nitrógeno y de fósforo que los materiales más lignificados, estos últimos son más resistentes a la descomposición (CALDATO et al., 2010).
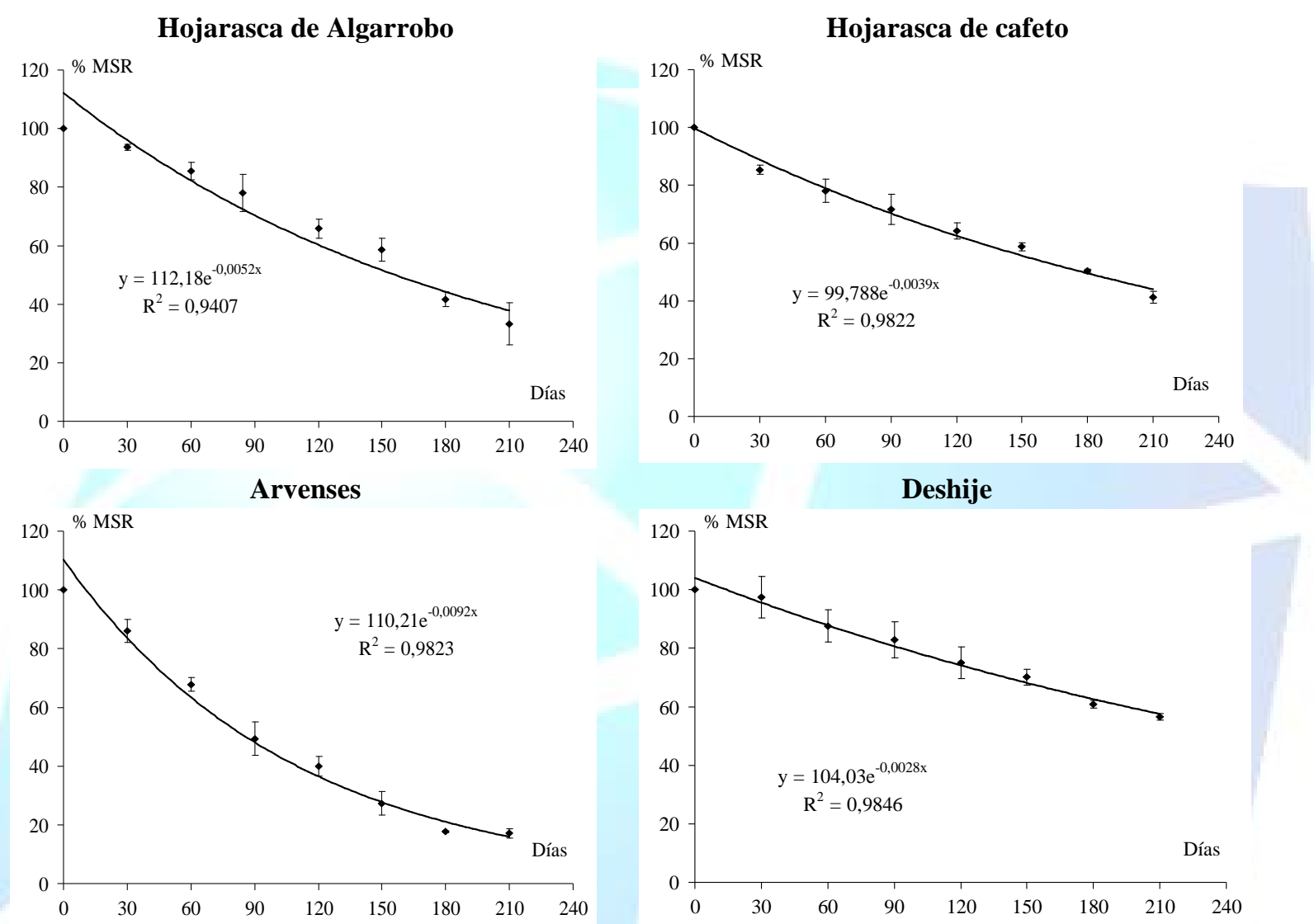

Figura 5. Tasa de descomposición de los diferentes restos estudiados. Medias de tres años de estudios. Sitio Tercer Frente. MSR. Masa seca remanente. Barras verticales: desviación estándar de las medias. Tercer Frente - (Tercer Frente, Santiago de Cuba), Cuba. .

Como resultado, la descomposición de los diferentes residuos estuvo en función de los materiales vegetales, su relación $\mathrm{C} / \mathrm{N}$ y el tiempo de duración del experimento. Esto concuerda con lo referido Poggiani y Schumacher (2004) y Pallardy (2008) quienes informaron que la forma y velocidad de descomposición de los restos están relacionadas principalmente con las condiciones climáticas, la relación $\mathrm{C} / \mathrm{N}$ y los microorganismos que efectúan el proceso.

Así, en los primeros días después de la caída de las hojas, ocurre mayor pérdida de compuestos solubles, de más fácil descomposición como los azúcares, aminoácidos y proteínas. Después de este período, este proceso se hace más lento por la presencia de materiales recalcitrantes como la celulosa (GOURETE, 2007). La fase final es llevada a cabo por hongos y bacterias del suelo y está más relacionada con los contenidos de ligninas presentes en los materiales. En términos generales, puede decirse que si la relación $\mathrm{C} / \mathrm{N}$ es mayor que 30, no existe una liberación inmediata 
del nitrógeno aprovechable en el suelo, por el contrario, si dicha relación es de 20 , algo del $\mathrm{N}$ se mineraliza y queda a disposición de la planta (CÓRDOVA, 2010).

\subsection{Liberación del nitrógeno}

Las hojas de cafeto, la hojarasca de algarrobo, las arvenses y los deshijes tuvieron inicialmente un proceso de inmovilización del $\mathrm{N}$ y su magnitud dependió del material evaluado. Las hojas de cafeto tuvieron un período de inmovilización entre los 30 - 50 días, a partir del cual se comenzó a liberar $\mathrm{N}$ al suelo de forma creciente hasta los 210 días que duró la investigación y alcanzó el 40\% de liberación (Figura 6). En Coffea arabica, Munguía (2003) encontró que a los 365 días de descomposición, la biomasa seca producida por el café transfirió al suelo el 64,8\% del N.

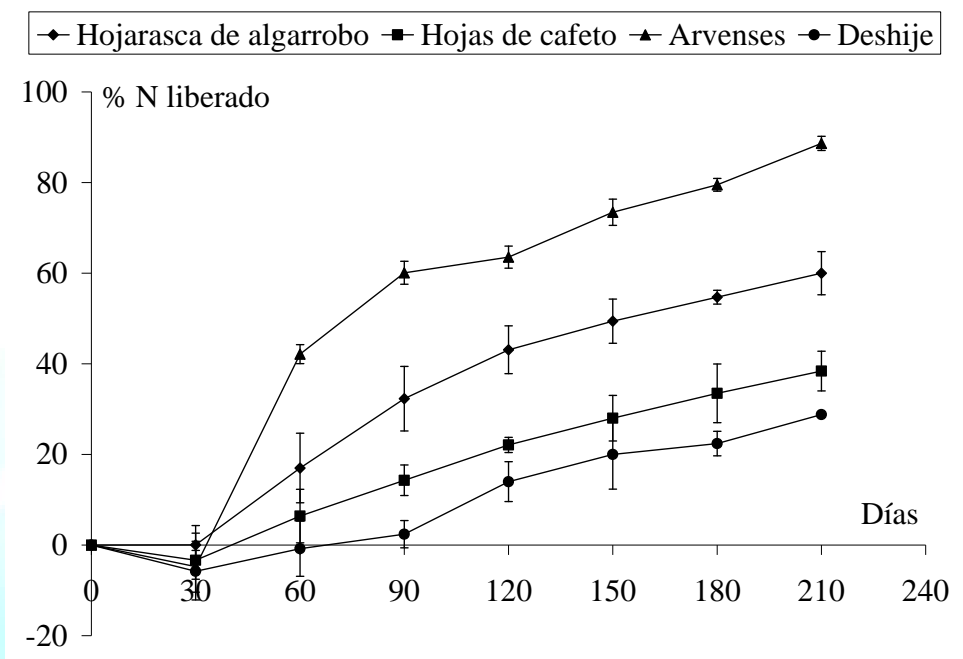

Figura 6. Flujo de $\mathrm{N}$ en diferentes restos del agroeocosistema cafetalero. Medias de los tres años evaluados. Tercer Frente. Barras verticales: desviación estándar de las medias Tercer Frente - (Tercer Frente, Santiago de Cuba) Cuba.

El período de inmovilización del $\mathrm{N}$ en la hojarasca de algarrobo estuvo por debajo de los 30 días, a partir de ese momento comenzó la liberación del $\mathrm{N}$ por parte de la hojarasca del algarrobo. A los 210 días se habían liberado $60 \%$ de nitrógeno que se encontraba en la masa seca.

Al respecto, Partielli et al. (2009) explicaran que la liberación de los nutrientes para el suelo ocurre en función de algunos factores, como la época del año (estación seca o lluviosa, verano o invierno), la localidad, la edad de la planta y la especie utilizada.

El nitrógeno en las arvenses, también se inmovilizó durante un mes, pero su liberación en el tiempo fue superior al resto de los materiales y llegó a los 210 días al $88 \%$ del contenido inicial. Los restos de los deshijes también se inmovilizaron en un período de 30 - 50 días, pero la magnitud de liberación de $\mathrm{N}$ fue la más lenta de todas, al alcanzar a los 210 días el 28\% de nitrógeno inicial contenido en la masa seca. Se encontraron diferencias en la tasa de descomposición de los residuos, los que perdieron peso en el orden siguiente: restos del deshierbe $\left(0.0092 \mathrm{k}^{-d i ́ a}{ }^{-1}\right)>$ hojarasca de algarrobo $\left(0.0052 \mathrm{k} . d i ́ a^{-1}\right)>$ hojas de café $\left(0.0039 \mathrm{k}^{-}\right.$día $\left.^{-1}\right)>$ restos del deshije del café $(0.0028$ k.día $\left.{ }^{-1}\right)$. El tiempo de vida media $\left(\mathrm{t}_{1 / 2}\right)$ varió entre las especies; así en las malezas el tiempo de vida fue el más bajo de los restos evaluados, con 76 días (Tabla 2). 
Tabla 2 - Coeficiente de descomposición $\left(\mathrm{k} \cdot \mathrm{dí}^{-1}\right)$, período de vida media y transferencia de $\mathrm{N}$ de los restos vegetales. Media de los tres años evaluados.Tercer Frente - (Tercer Frente, Santiago de Cuba), Cuba.

\begin{tabular}{|c|c|c|}
\hline Restos vegetales & $\begin{array}{c}\text { Coeficiente de } \\
\text { descomposición }\end{array}$ & $\begin{array}{c}\text { Período medio de vida } \\
\mathbf{t}_{1 / 2} \text { (días) }\end{array}$ \\
\hline Hojas de cafeto & 0,0039 & 203,02 \\
\hline Hojarasca de algarrobo & 0,0052 & 188,54 \\
\hline Arvenses & 0,0092 & 76,3 \\
\hline Restos de deshijes & 0,0028 & 252,99 \\
\hline
\end{tabular}

Los restos del algarrobo tuvieron un período de vida media de 188 días, inferior a los resultados obtenidos con la especie Inga sp. utilizada como sombra para el cafeto en Brasil, con un tiempo de vida media de su hojarasca de 236 días (GOURETE, 2007), y a lo reportado para la descomposición de la hojarasca de la selva mixta del sur de Brasil, donde se encontró un tiempo de vida media de 3.89 años (CALDATO et al., 2010).

En el caso de las hojas café, la vida media de sus residuos fue de 203 días aproximadamente, mientras que los residuos del deshije no perdieron el $50 \%$ de su peso durante los días que duró la investigación.

\section{CONCLUSIONES}

Se encontraron altos aportes de biomasa anual por los diferentes componentes del agroecosistema, todos ellos con importantes retornos de $\mathrm{N}$ al suelo. Las hojas de cafeto, la hojarasca de algarrobo, las arvenses y los deshijes tuvieron inicialmente un proceso de inmovilización del $\mathrm{N}$ hasta 30 dias y posteriormente comenzaron a liberar el $\mathrm{N}$ de su biomasa, pero su magnitud dependió del material evaluado. Se encontraron diferencias en la tasa de descomposición de los residuos dentro del agroecosistema cafetalero, con un tiempo de vida media $\left(\mathrm{t}_{1 / 2}\right)$ que varió entre las especies.

\section{AGRADECIMIENTOS}

A los colegas del Instituto de Investigaciones Agroforestales, UCTB Tercer Frente, el Instituto Nacional de Ciencias Agrícolas y al Centro de Estudos Ambientais (CEA) de la Universidad Estadual Paulista (UNESP), por la asesoría brindada en el trabajo.

\section{REFERENCIAS}

ANDERSON, J.M.; INGRAM, J.S.I. Tropical soil fertility: a handbook of methods. Cab International, 2nd edition, UK, 1996. p. 36-40.

ARELLANO, R.J.; PAOLINI, L.V.; MORA, E. Producción y descomposición de la hojarasca en tres agroecosistemas de café en el estado Trujillo, Venezuela. Revista Forestal Venezolana, Merida, Venezuela, v.48, n.1, p. 7-14, 2004.

CALDATO, S.L.; FLOSS, A.; SERAFINI F.; ELZIRA C. Litter production and decomposition in a mixed araucaria forest in the south of Brazil. Bosque, Valdivia, v.31 n.1: p. 3-8, 2010. 
CARDONA, D.A. Caracterización de la fertilidad del suelo y ciclo de nutrientes en monocultivo de café (Coffea arabica L.) y bajo sombrío de guamo (Inga spp.). 2004. $178 \mathrm{f}$. Disertación (Maestrado) - Facultad de Medioambiente y Recursos Naturales, Universidad Distrital Francisco José de Caldas, Bogota, 2004.

CARDONA, D.; SADEGHIAN, S. Ciclo de nutrientes y actividad microbiana en cafetales a libre exposición solar y con sombrío de Inga spp. Cenicafé, Colombia, Bogota, v.56, n. 2, p. 127-141, 2005.

CÓRDOVA, G. Mineralización de nitrógeno de diferentes abonos orgánicos. 2010. 85 f. Disertación (Maestrado en Ciencias) - Colegio de Posgraduados. Institución de Enseñanza e Investigación en Ciencias Agrícolas, Campus Tabasco. H. Cárdena, Tabasco, México., 2010.

DÍAZ, W.; VÁZQUEZ, E.G.; MOLINA, R.R. Manejo de la poda quinquenal de Coffea canephora Pierre ex Froehner. Café y Cacao, Santiago de Cuba, Cuba, v.8, n.2, p. 15-23, 2007.

GARBISU, C.; BECERRIL, J.M.; EPELDE, L.; ALKORTA I. Bioindicadores de la calidad del suelo: herramienta metodológica para la evaluación de la eficacia de un proceso fitorremediador. Ecosistema, España, v.16, p. 1697-2473, 2007.

GOURETE, D.E. Ciclagem de nutrientes por árvores em sistemas agroflorestais na Mata Atlântica. 2007; 132 f. Disertación (Maestrado) - Facultad de Agronomia, Universidad Federal de Viçosa. Viçosa, Minas Gerais, 2007.

JIMÉNEZ, A.; FARFÁN, F.; MORALES, C. Descomposición y transferencia de nutrientes de Cajanus cajan, Crotalaria juncea y Tephrosia candida como abonos verdes en cafetales. Cenicafé, Colombia, v.56, n.3, p. 216-236, 2005.

MUNGUÍA, R. Tasas de descomposición y liberación de nutrientes de la hojarasca de Eucalyptus deglupta, Coffea arabica y de hojas verdes de Erythrina poeppigiana solas y en mezclas. 2003, 98 f. Disertación (Maestrado). Centro de Investigación Agricolas - CIAT, Turrialba, Colombia, 2003.

PÉREZ, D.; SOUZA, O. Producción, descomposición y liberación de nutrientes de la hojarasca bajo café a pleno sol y con sombra de Gliricidia sepium (Jacq) en Carazo, Nicaragua. 2008, 65 f. Trabajo de diploma. Facultad de Recursos Naturales y del Ambiente, Universidad Nacional Agraria. Managua. Nicaragua, 2008.

PEREZ, A.; BUSTAMANTE, C.; MARTIN, G., RIVERA R., VIÑALS, R. Fertilización nitrogenada después de la poda del cafeto robusta en Cambisoles. Pesquisa Agropecuária Brasileira, Brasília, DF, v. 46, n. 8, p. 935-943, 2011.

PÉREZ, A.; CASTAÑEDA, E.; LOZANO, S.; BUSTAMANTE C.; RIVERA, R.; RODRÍGUEZ, G.; MARTÍN, G.; ROBLES, G.; ACOSTA, G.; FERNÁNDEZ, A. Foliar Analysis as an Estimate on the Nutritional State of Conilon Coffee Plantations on Cambisoils. Journal of Life Sciences, David Plublishs, USA, v.9, n.2, p. 181-187, 2014a. 
PEREZ, A.; BUSTAMANTE, C.; MARTÍN, G.; RIVERA, R. Efecto de la fertilización nitrogenada en el cafeto conilon sobre el rendimiento y algunos indicadores de calidad de suelos Cambisoles de Cuba. Holos Environment, Rio Claro, Sao Paulo, v.14, n.1, p. 49-55, 2014 b.

PARTIELLI L.; DUARTE H.; RIBEIRO F. Cultivo com plantas de cobertura: alternativa viável. A lavoura, Instituto Agronómico do Parana, Brasil, v.112, n. 671, p. 42-47, 2009.

PALLARDY, S.G. Physiology of woody plants. $3^{\text {rd }}$ Ed. New York, USA. Elsevier, 2008. 454 p.

PHILPOTT, S.M.; PERFECTO, I.; VANDERMEER, J. Effects of management system and season on arboreal ant diversity and abundance in coffee agroecosystems. Revista Brasileira do Botanica, São Paulo, Brasil, v.15, p.139-155, 2006.

POGGIANI, F.; SCHUMACHER, M.V. Nutrient cycling in native forests. In: Gonçalves, J.L.M.; Benedetti, V. (Eds). Forest nutrition and fertilization. Piracicaba, Brasil. IPEF, 2004. p. 287-305.

QUINTERO, J.S.; ATAROFF, M. Contenido y flujos de nitrógeno en la biomasa y hojarasca de un cafetal a plena exposición solar en Los Andes venezolanos. Revista Facultad Agronomía, Venezuela, v.15, p. 501-514, 1998. 